\title{
Minimum clinical important difference for resilience scale specific to cancer: a prospective analysis
}

\author{
Zeng Jie Ye ${ }^{1^{*}+}\left(\mathbb{D}\right.$, Zhang Zhang ${ }^{2 \dagger}$, Ying Tang ${ }^{3}$, Jian Liang ${ }^{4}$, Xiao Ying Zhang ${ }^{5}$, Guang Yun $\mathrm{Hu}^{6}$, Zhe Sun ${ }^{7}$,
} Mu Zi Liang ${ }^{8}$ and Yuan Liang Y ${ }^{9}$

\begin{abstract}
Background: The minimum clinical important differences (MCIDs) of resilience instruments in patients with cancer have not been comprehensively described.This study was designed to evaluate MCIDs of 10-item and 25-item resilience scales specific to cancer (RS-SC-10 and RS-SC-25).

Methods: From June 2015 to December 2018, RS-SCs were longitudinally measured in 765 patients with different cancer diagnoses at baseline (T0) and 3 months later (T1). The EORTC QLQ-C30, Connor-Davidson Resilience Scale, Hospital Anxiety and Depression Scale, and Allostatic Load Index were measured concurrently as anchors. Anchorbased methods (linear regression, within-group), distribution-based methods(within-group), and receiver operating characteristic curves (ROCs, within-subject) were performed to evaluate the MCIDs.
\end{abstract}

Results: 623 of 765 (84.1\%) patients had paired RS-SCs scores. Moderate correlations were identified between the change in RS-SCs and change in anchors ( $r=0.38-0.44$, all $p<0.001)$. Linear regression estimated +8.9 and -6.7 as the MCIDs of RS-SC-25, and +3.4 and -2.5 for RS-SC-10. Distribution-based methods estimated +9.9 and -9.9 as the MCIDs of RS-SC-25, and +4.0 and -4.0 for RS-SC-10. ROC estimated +5.5 and -4.5 as the MCIDs of RS-SC-25, and +2.0 and -1.5 for RS-SC-10.

Conclusions: The most reliable MCID is around 5 points for RS-SC-25 and 2 points for RS-SC-10. RS-SCs are more responsive to the worsening status of resilience in patients with cancer and these estimates could be useful in future resilience-based intervention trials.

Keywords: Resilience, Nursing, Cancer, RS-SC-25, RS-SC-10, Minimum clinical important difference, Anchor-based, Distribution-based, ROC

\section{Introduction}

In China, about 4.5 million people were diagnosed with cancer and 2.9 million people died from it in 2019 [1].The mortality of cancer has been lowered owing to advances

\footnotetext{
*Correspondence: zengjieye@qq.com

${ }^{\dagger}$ Zeng Jie Ye and Zhang Zhang are contributed equally to this work and should be considered co-first authors

${ }^{1}$ Guangzhou University of Chinese Medicine, Guangzhou 510006, Guangdong Province, China

Full list of author information is available at the end of the article
}

in medical technology, and cancer is increasingly treated as a chronic disease [2]. Thus, helping cancer survivors rehabilitate from the traumatic event is a topic receiving enhanced interest in cancer research. Resilience is defined as one's ability to 'bounce back' from adversity and is a salient indicator of patients' quality of life and psychosocial functions [3, 4]. However, no resilience scales have been formulated to be specific to patients with cancer, and the application of generic resilience instruments among cancer-specific populations has been criticized [5]. To address this issue, we developed a original author(s) and the source, provide a link to the Creative Commons licence, and indicate if changes were made. The images or other third party material in this article are included in the article's Creative Commons licence, unless indicated otherwise in a credit line to the material. If material is not included in the article's Creative Commons licence and your intended use is not permitted by statutory regulation or exceeds the permitted use, you will need to obtain permission directly from the copyright holder. To view a copy of this licence, visit http://creativecommons.org/licenses/by/4.0/. The Creative Commons Public Domain Dedication waiver (http://creativeco mmons.org/publicdomain/zero/1.0/) applies to the data made available in this article, unless otherwise stated in a credit line to the data. 
25-item Resilience Scale Specific to Cancer (RS-SC-25) as an aid for nurses, physicians and social workers to assess the resilience levels of patients [6]; RS-SC-25 was then validated based on classic theory test, item response theory, and resilience-related empirical research $[7,8]$. The RS-SC-25 has Chinese and English versions and consists of five domains (generic element, benefit finding, support and coping, hope for the future, and meaning for existence); higher scores indicate higher resilience levels [6]. RS-SC-25 has good psychometric properties and could be used as a means of establishing a symptomatic threshold to guide the initiation of psychosocial or pharmacological intervention. A short 10-item version (RS-SC-10) with higher discriminative items has been developed according to multidimensional item response theory (MIRT) to reduce the scale burden on the patients; its potential in outpatient wards and communities has been confirmed [9]. However, the responsiveness of both RS-SCs (RS-SC25 and RS-SC-10), that is, its ability to measure changes over time, has not been evaluated, raising concerns on the use of this instrument in routine practice. To enable an appropriate assessment of resilience-related changes in patients with cancer, the minimum clinically important difference (MCID), which is the smallest change in score that patients perceive as beneficial or detrimental, is important to the clinical interpretation of scale data, especially for resilience-related interventions in different settings $[10,11]$. However, to our knowledge, the MCID of resilience instruments in patients with cancer have not been comprehensively described. Thus, the current study was designed to calculate the MCIDs of RS-SCs using distribution- and anchor-based methods. Based on previous findings $[4,6-8,12-14]$, resilience was strongly associated with anxiety, depression, quality of life, and Allostatic Load Index. Thus, we postulated the following: (1) RS-SCs would be significantly associated with established scales, namely, European Organization for Research and Treatment of Cancer Quality of Life Questionnaire (EORTC QLQ-C30, measuring quality of life), Connor-Davidson Resilience Scale (CD-RISC, measuring generic resilience), Hospital Anxiety and Depression Scale (HADS, measuring anxiety and depression), and Allostatic Load Index (ALI, measuring physiological load) in this study; and (2) changes in RS-SCs would be significantly associated with changes in established scales.

\section{Methods}

\section{Sample/participants}

Participants were enrolled from six hospitals in Guangdong and Heilongjiang Provinces between June 2015 and December 2018; each had a confirmed diagnosis of cancer based on biopsy and medical imaging. All participants had to fulfill the same inclusion/exclusion criteria, which were detailed as follows, inclusion: (1) aged 18-65 years, (2) had the ability to communicate in Mandarin or Cantonese fluently, and (3) receiving active treatment; exclusion: (1) misdiagnosed with cancer, (2) cannot communicate in Mandarin or Cantonese fluently, and (3) unwilling to participate in the study. Patients were all derived from a big project named as Be Resilient to Cancer. Informed consent was obtained, and the Human Research Ethics Committee approved the present study (registration number: 2016KYTD08).

\section{Data collection}

This prospective study was conducted between June 2015 and December 2018. RS-SCs, EORTC QLQ-C30, CDRISC, and HADS were administered to 765 patients at baseline (T0), whereas ALI was conducted in 275 patients treated in the six hospitals. Three months later (T1), the same instruments were administered again.

\section{Measures \\ RS-SC}

The original RS-SC is a 25 -item resilience instrument specific to cancer (RS-SC-25) that has the five domains of generic element, benefit finding, support and coping, hope for the future, and meaning for existence [6]. A 10-item RS-SC (RS-SC-10) was developed later by MIRT analysis [9]. The two scales are both rated based on a fivepoint Likert scale, with higher scores indicating higher resilience levels. Scores for RS-SC-25 range from 25 to 125, and for RS-SC-10, from 10 to 50. The Cronbach's $\alpha$ and test-retest reliability of RS-SC-25 are 0.83 and 0.87 , respectively $[6,9,15]$. The Cronbach's $\alpha$ of RS-SC-10 is 0.86 . RS-SC-25 and RS-SC-10 are attached in the Additional file 1 (Additional file 1: Tables S1 and S2).

\section{EORTC QLQ-C30}

EORTC QLQ-C30 is a 30-item quality of life (QoL) instrument specific to cancer, including five functional dimensions, three symptom dimensions, a global health status, some additional symptom items reported by patients with cancer, and perceived financial impact of cancer [16]. The raw score of each dimension can be converted to a score ranging from 0 to 100 according to the manual, with higher scores indicating better functional ability or increased distress in the symptom items [17]. In addition, Item 30 measuring the global health status was used as an anchor in this study (raw score ranging from 1 to 7$)$. The Cronbach's $\alpha$ of the Chinese version of EORTC QLQ-C30 ranges from 0.78 to 0.93 in different dimensions. 


\section{CD-RISC}

The Chinese version of CD-RISC is a 25 -item generic resilience instrument with the three dimensions of tenacity, strength, and optimism [18]. Additional two short non-dimensional versions of CD-RISC (2- and 10-item, named CD-RISC-2 and CD-RISC-10, respectively) were developed later. These three scales are all rated based on a five-point Likert scale with higher scores indicating higher resilience levels (ranging from 0 to $8,0-40,0-100$, respectively). CD-RISC-10 was used as an anchor in this study. Cronbach's $\alpha$ s of $0.83,0.79$, and 0.85 are identified for CD-RISC-25, CDRISC-2, and CD-RISC-10, respectively $[19,20]$.

\section{HADS}

The Chinese version of HADS is a 14-item emotional distress-screening tool with seven items for anxiety and seven items for depression [21]. The instrument is scored on a five-point scale with higher scores indicating worse emotional functions (ranging from 0 to 56). HADS was used as an anchor in this study. The Cronbach's $\alpha$ of HADS is 0.91 .

\section{ALI}

ALI is a validated composite index measuring 14 indicators from different physiological systems, such as the functions of the sympathetic nervous system, parasympathetic nervous system, and hypothalamic pituitary adrenal. ALI scores range from 0 to 14 , with higher scores indicating higher allostatic load. Similar approaches to conceptualize physical allostatic load have been applied [22-25]. The Cronbach's $\alpha$ of ALI has not been evaluated.

\section{Data analysis}

First, at T0, Pearson's $r$ correlation coefficients were used to measure the correlations between RS-SCs (RS-SC-25 and RS-SC-10) and anchors in patients with different cancer diagnoses [26, 27]. Fisher's z-transformation was applied to approximate the variance-stabilizing transformation for Pearson's $r$ correlation coefficients when RS-SCs and anchors followed a bivariate normal distribution, and determine the $95 \%$ confidence intervals (CIs) for the Pearson's $r$ correlation coefficients $[28,29] . r<0.3$, $0.3 \leq \mathrm{r} \leq 0.5, \mathrm{r}>0.5$ were defined as weak, moderate and strong coefficients, respectively.

Second, from T0 to T1, Pearson's $r$ correlation coefficients $(95 \% \mathrm{CI})$ were performed again to estimate the correlations between change in RS-SCs and change in anchors, in order to select suitable anchors. The correlation coefficients should be more than 0.30 , as recommended [11].
Third, from T0 to T1, linear regression (95\%CI, within-group) were calculated to compare changes in RS-SCs against different anchors. As for linear regression, change in RS-SC score (independent variable) was anchored against change in EORTC QLQ-C30, CDRISC, and HADS score (dependent variables), respectively. In addition, Cohen effect size (ES) was also calculated [30]. $\mathrm{ES}<0.3,0.3 \leq \mathrm{ES} \leq 0.8$, ES $>0.8$ were defined as small, medium, and large effect. For the distribution-based estimation (within-group) of MCIDs, we calculated the $20 \%$ (0.2 SD), 30\% (0.3 SD), and 50\% (0.5 SD) SD [31]; standard error of measurement (SEM) [32]; and minimal detectable change (MDC) for the 90\% CI $\left(\mathrm{MDC}_{90}\right)$ and $95 \% \mathrm{CI}\left(\mathrm{MDC}_{95}\right)$ [10].

At last, from $\mathrm{T} 0$ to $\mathrm{T} 1$, receiver operating characteristic curves (ROCs, within-subject) analysis were performed. Changes in the cut-off of RS-SCs that best discriminated between patients who increased or decreased their resilience levels by the established MCIDs in the EORTC QLQ-C30 (1-point change in global health status, QoL-GHS) [33], CD-RISC-10 (3-point change) [34], and HADS (1.5-point change for anxiety and depression each) were defined as showing MCID [35]. The area under curve (AUC) and Youden index were adapted with equal weighting given to sensitivity and specificity [36]. $P<0.05$ was recognized as statistically significant for all the data analysis. All data analyses were performed using SPSS 21 (IBM, USA).

\section{Results \\ Demographics}

623 of 765 (84.1\%) patients had paired RS-SCs scores. Breast, gastric, and lung cancer were the three most common cancer diagnoses, constituting 33.5, $20.1,13.3 \%$ of all cases, respectively. One hundred fortytwo patients were excluded from the analysis for the following reasons:unwillingness $(N=37)$, busy schedule $(N=24)$, lost to follow-up $(N=55)$, and incomplete responses $(N=26)$. No significant demographic difference was identified between the included and the excluded. The baseline characteristics of the patients are presented in Table 1.

\section{Correlations between RS-SCs and anchors at baseline (T0)}

The means (SD) of the RS-SCs and anchors are described in Table 2. Acceptable correlations (from 0.38 to $0.73, P<0.001$ ) were identified between RSSCs and anchors. In general, the correlations between RS-SC-10 and established scales were stronger than those between RS-SC-25 and external indicators. 
Table 1 Characteristics of patients in the analysis of RS-SCs $(N=765)$

\begin{tabular}{|c|c|c|c|c|c|c|}
\hline Characteristics (\%) & Lung cancer & Gastric cancer & Colon-rectal cancer & Liver cancer & Leukemia & Breast cancer \\
\hline No & $102(13.3)$ & $154(20.1)$ & $98(12.8)$ & $81(10.6)$ & $74(9.7)$ & $256(33.5)$ \\
\hline \multicolumn{7}{|l|}{ Sex } \\
\hline Female & $29(28.4)$ & $73(47.4)$ & $50(51.0)$ & $29(35.8)$ & $34(45.9)$ & $256(100.0)$ \\
\hline Man & $73(71.6)$ & $81(52.6)$ & $48(49.0)$ & $52(64.2)$ & $40(54.1)$ & $0(0.0)$ \\
\hline Age (years, SD) & $44.7(17.1)$ & $57.1(16.9)$ & $53.1(14.8)$ & $46.8(13.9)$ & $39.1(16.9)$ & $39.3(14.7)$ \\
\hline $\begin{array}{l}\text { Time since first confirmed diagnosis } \\
\text { (months, SD) }\end{array}$ & $14.1(12.0)$ & $17.8(8.7)$ & $20.9(12.5)$ & $9.9(10.1)$ & $8.1(6.9)$ & $5.2(6.1)$ \\
\hline Duration of treatment (months, SD) & $6.7(5.5)$ & $11.9(8.0)$ & $15.9(9.2)$ & $6.3(5.4)$ & $7.1(8.0)$ & $4.6(7.4)$ \\
\hline \multicolumn{7}{|l|}{ Education level } \\
\hline Middle school or lower & $58(56.9)$ & $91(59.1)$ & $52(53.1)$ & $42(51.9)$ & $43(58.1)$ & $112(43.8)$ \\
\hline High school or higher & $44(43.1)$ & $63(40.9)$ & $46(46.9)$ & $39(48.1)$ & $31(41.9)$ & $144(56.2)$ \\
\hline \multicolumn{7}{|l|}{$\begin{array}{l}\text { Family income } \\
\text { (RMB per month) }\end{array}$} \\
\hline$<5000$ & $47(46.1)$ & $85(55.2)$ & $49(50.0)$ & $39(48.1)$ & $34(45.9)$ & $97(37.9)$ \\
\hline $5000-10,000$ & $28(27.5)$ & $41(26.6)$ & $26(26.5)$ & $32(39.5)$ & $28(37.8)$ & $116(45.3)$ \\
\hline$>10,000$ & $27(26.4)$ & $28(18.2)$ & $23(23.5)$ & $10(12.4)$ & $12(16.2)$ & $43(16.8)$ \\
\hline \multicolumn{7}{|l|}{ Marital status } \\
\hline Married & $85(83.3)$ & $124(80.5)$ & 77 (78.6) & $62(76.5)$ & $58(78.4)$ & $157(61.3)$ \\
\hline Single or other & $17(16.7)$ & $30(19.5)$ & $21(21.4)$ & $19(23.5)$ & $16(21.6)$ & $99(38.7)$ \\
\hline \multicolumn{7}{|l|}{ Religious beliefs } \\
\hline Yes & 18 (17.6) & $41(26.6)$ & 28 (28.6) & $28(34.6)$ & $21(28.4)$ & 65 (25.4) \\
\hline None & $84(82.4)$ & $113(73.4)$ & $70(71.4)$ & $53(65.4)$ & $53(71.6)$ & $191(74.6)$ \\
\hline \multicolumn{7}{|l|}{ Employment status } \\
\hline Employment & $54(52.9)$ & $76(49.4)$ & $43(43.9)$ & $30(37.0)$ & $38(51.4)$ & $162(63.3)$ \\
\hline Unemployment & $48(47.1)$ & $78(50.6)$ & $55(56.1)$ & $51(63.0)$ & $36(48.6)$ & $94(36.7)$ \\
\hline \multicolumn{7}{|l|}{ Stage of cancer } \\
\hline I & $15(14.7)$ & $19(12.3)$ & $21(21.4)$ & $15(18.5)$ & NA & $77(30.1)$ \\
\hline$\|$ & $29(28.4)$ & $71(46.1)$ & $23(23.5)$ & $28(34.6)$ & NA & $87(34.0)$ \\
\hline III & $41(40.2)$ & $46(29.9)$ & $32(32.7)$ & $25(30.9)$ & NA & $74(28.9)$ \\
\hline IV & $17(16.7)$ & $18(11.7)$ & $22(22.4)$ & $13(16.0)$ & NA & $18(7.0)$ \\
\hline \multicolumn{7}{|l|}{ Type of therapy } \\
\hline Chemo & $41(40.2)$ & $63(40.9)$ & 45 (45.9) & $57(70.4)$ & $56(75.7)$ & $146(57.0)$ \\
\hline Radiation & $23(22.5)$ & $32(20.8)$ & $30(30.6)$ & $31(38.3)$ & $13(17.6)$ & $54(21.1)$ \\
\hline Surgery & $22(21.6)$ & $84(54.5)$ & $57(58.2)$ & $37(45.7)$ & $10(13.5)$ & $138(53.9)$ \\
\hline \multicolumn{7}{|l|}{ Combordities } \\
\hline None & $25(34.3)$ & $55(35.7)$ & $26(26.5)$ & 25 (30.9) & $40(54.1)$ & $152(59.4)$ \\
\hline One & $36(35.3)$ & $62(40.3)$ & $41(41.8)$ & 38 (46.9) & $24(32.4)$ & $83(32.4)$ \\
\hline Two or more & $41(40.2)$ & $37(24.0)$ & $31(31.7)$ & $18(22.2)$ & $10(13.5)$ & $21(8.2)$ \\
\hline
\end{tabular}

NA not available

\section{Correlations between changes in RS-SCs and changes in anchors from T0 to T1}

The correlations between change in RS-SCs and change in anchors are presented in Table 3. Changes in QoLGHS $(r=0.38(0.28$ to 0.47$), \mathrm{ES}=0.26, P<0.001)$, CDRISC-10 $\quad(0.44(0.35$ to 0.52$), \quad E S=0.21, \quad P<0.001)$, HADS-A $\quad(r=-0.35(-0.44$ to -0.25$), \quad \mathrm{ES}=0.19$, $P<0.001)$, HADS-D $(r=-0.41(-0.50$ to -0.31$)$, $\mathrm{ES}=0.23, P<0.001)$, significantly correlated with changes in RS-SC-25 except for ALI ( $r=0.19(-0.29$ to -0.08$)$, $\mathrm{ES}=0.11, P=0.001)$. Similar results were identified between RS-SC-10 and anchors. Thus, ALI could not be used as an anchor in the MCID analysis.

\section{MCID estimation of RS-SCs from T0 to T1}

Using the distribution-based indicator of SEM, the mean MCIDs of RS-SC-10 and RS-SC-25 were \pm 3.51 and \pm 9.18 , respectively. Using the distribution-based 
Table 2 Pearson's correlation coefficients between RS-SCs and potential anchors at baseline $\left(T_{0}, N=765\right)$

\begin{tabular}{|c|c|c|c|c|c|c|c|c|}
\hline Variables & Mean (SD) & Score range & Item No & Cronbach a & RS-SC-10 & RS-SC-25 & & \\
\hline & & & & & $r(95 \% \mathrm{Cl})$ & $P$ & $r(95 \% \mathrm{Cl})$ & $P$ \\
\hline QoL GHS & $55.73(20.62)$ & $0-100$ & 1 & NA & 0.54 (0.48 to 0.59$)$ & $<0.001$ & 0.51 (0.45 to 0.57 ) & $<0.001$ \\
\hline QoL PF & $64.86(29.37)$ & $0-100$ & 5 & 0.85 & 0.39 (0.32 to 0.45$)$ & $<0.001$ & 0.38 (0.31 to 0.44$)$ & $<0.001$ \\
\hline QoL RF & 47.89 (19.89) & $0-100$ & 2 & 0.79 & 0.56 (0.50 to 0.61$)$ & $<0.001$ & 0.53 (0.47 to 0.58 ) & $<0.001$ \\
\hline QoL EF & $40.54(16.91)$ & $0-100$ & 4 & 0.86 & 0.65 (0.60 to 0.69$)$ & $<0.001$ & 0.60 (0.55 to 0.65 ) & $<0.001$ \\
\hline QoL CF & $58.97(21.12)$ & $0-100$ & 2 & 0.74 & 0.53 (0.47 to 0.58 ) & $<0.001$ & 0.52 (0.46 to 0.57 ) & $<0.001$ \\
\hline QoL SF & $68.78(30.66)$ & $0-100$ & 2 & 0.76 & 0.61 (0.56 to 0.66 ) & $<0.001$ & 0.57 (0.51 to 0.62 ) & $<0.001$ \\
\hline CD-RISC-25 & $46.34(21.88)$ & $0-100$ & 25 & 0.83 & 0.64 (0.59 to 0.68 ) & $<0.001$ & 0.59 (0.54 to 0.64 ) & $<0.001$ \\
\hline CD-RISC-10 & $18.85(6.93)$ & $0-40$ & 10 & 0.85 & 0.73 (0.69 to 0.76 ) & $<0.001$ & 0.67 (0.62 to 0.71$)$ & $<0.001$ \\
\hline CD-RISC-2 & $3.48(1.46)$ & $0-8$ & 2 & 0.74 & 0.51 (0.45 to 0.57 ) & $<0.001$ & 0.48 (0.42 to 0.54 ) & $<0.001$ \\
\hline HADS total & 18.08 (6.95) & $0-42$ & 14 & 0.80 & $-0.59(-0.64$ to -0.54$)$ & $<0.001$ & $-0.56(-0.61$ to -0.50$)$ & $<0.001$ \\
\hline HADS-A & $8.43(3.12)$ & $0-21$ & 7 & 0.84 & $-0.46(-0.52$ to -0.40$)$ & $<0.001$ & $-0.45(-0.51$ to -0.39$)$ & $<0.001$ \\
\hline HADS-D & $9.65(3.89)$ & $0-21$ & 7 & 0.87 & $-0.68(-0.72$ to -0.64$)$ & $<0.001$ & $-0.59(-0.64$ to -0.54$)$ & $<0.001$ \\
\hline $\mathrm{ALI}$ & $5.86(2.87)$ & $0-14$ & 14 & NA & $-0.45(-0.51$ to -0.39$)$ & $<0.001$ & $-0.43(-0.49$ to -0.36$)$ & $<0.001$ \\
\hline
\end{tabular}

RS-SC Resilience Scale Specific to Cancer, HADS Hospital Anxiety and Depression Scale, QoL Quality of Life, GHS Global Health Status, PF Physical Function, RF Role Function, EF Emotion Function, CF Cognitive Function, SF Social Function, CD-RISC Connor-Davidson Resilience Scale, ALI Allostatic Load Index, NA not available

Table 3 Pearson's correlations between change in RS-SCs and change in potential anchors at 3-month $\left(T_{1}, N=643\right)$

\begin{tabular}{|c|c|c|c|c|c|c|}
\hline \multirow[t]{2}{*}{ Variables } & \multirow[t]{2}{*}{ Changes $(95 \% \mathrm{Cl})$} & \multirow[t]{2}{*}{ Effect size } & \multicolumn{2}{|l|}{ RS-SC-10 } & \multicolumn{2}{|l|}{ RS-SC-25 } \\
\hline & & & $r(95 \% \mathrm{Cl})$ & $\mathbf{P}$ & $r(95 \% \mathrm{Cl})$ & $\mathbf{P}$ \\
\hline QoL GHS & $5.13(3.34$ to 7.20$)$ & 0.26 & 0.38 (0.28 to 0.47) & $<0.001$ & $0.32(0.22$ to 0.41$)$ & $<0.001$ \\
\hline QoLPF & 1.21 (0.96 to 1.51$)$ & 0.05 & 0.15 (0.04 to 0.26$)$ & 0.092 & 0.18 (0.08 to 0.28$)$ & 0.004 \\
\hline QoL RF & $2.52(2.30-2.79)$ & 0.16 & 0.31 (0.21 to 0.41$)$ & $<0.001$ & 0.26 (0.16 to 0.35$)$ & $<0.001$ \\
\hline QoLEF & 4.04 (3.63 to 4.39$)$ & 0.23 & 0.39 (0.29 to 0.48 ) & $<0.001$ & 0.33 (0.23 to 0.42 ) & $<0.001$ \\
\hline QoL CF & 0.55 (0.37 to 0.74$)$ & 0.03 & 0.16 (0.05 to 0.27$)$ & 0.061 & 0.17 (0.07 to 0.27$)$ & 0.029 \\
\hline QoL SF & $3.51(3.19-3.81)$ & 0.13 & 0.32 (0.22 to 0.42$)$ & $<0.001$ & $0.32(0.22$ to 0.41$)$ & $<0.001$ \\
\hline CD-RISC-25 & 6.12 (5.54 to 6.81$)$ & 0.27 & 0.36 (0.26 to 0.45$)$ & $<0.001$ & 0.34 (0.24 to 0.43$)$ & $<0.001$ \\
\hline CD-RISC-10 & 1.22 (0.97 to 1.54) & 0.21 & 0.44 (0.35 to 0.52) & $<0.001$ & $0.38(0.29$ to 0.47$)$ & $<0.001$ \\
\hline CD-RISC-2 & 0.24 (0.18 to 0.29 ) & 0.19 & 0.32 (0.22 to 0.42$)$ & $<0.001$ & 0.29 (0.19 to 0.38 ) & $<0.001$ \\
\hline HADS total & $-1.26(-1.45$ to -1.07$)$ & -0.22 & $-0.38(-0.47$ to -0.28$)$ & $<0.001$ & $-0.34(-0.43$ to -0.24$)$ & $<0.001$ \\
\hline HADS-A & $-0.51(-0.64$ to -0.38$)$ & -0.19 & $-0.35(-0.44$ to -0.25$)$ & $<0.001$ & $-0.30(-0.39$ to -0.20$)$ & $<0.001$ \\
\hline HADS-D & $-0.75(-0.89$ to -0.60$)$ & -0.23 & $-0.41(-0.50$ to -0.31$)$ & $<0.001$ & $-0.36(-0.45$ to -0.27$)$ & $<0.001$ \\
\hline ALI & $-0.27(-0.32$ to -0.22$)$ & -0.11 & $-0.19(-0.29$ to -0.08$)$ & 0.001 & $-0.20(-0.30$ to -0.10$)$ & $<0.001$ \\
\hline
\end{tabular}

Italics rows indicate suitable anchors

RS-SC Resilience Scale Specific to Cancer, HADS Hospital Anxiety and Depression Scale, QoL Quality of Life, GHS Global Health Status, PF Physical Function, RF Role Function, EF Emotion Function, CF Cognitive Function, SF Social Function, CD-RISC Connor-Davidson Resilience Scale, ALI Allostatic Load Index

Table 4 Distribution-based MCID for RS-SCs at baseline $\left(T_{0}, N=765\right)$ and 3-month $\left(T_{1}, N=643\right)$

\begin{tabular}{|c|c|c|c|c|c|c|c|c|c|c|c|c|}
\hline \multirow[t]{2}{*}{ Scales } & \multicolumn{6}{|l|}{ TO } & \multicolumn{6}{|l|}{ T1 } \\
\hline & S.E.M & MDC90 & MDC95 & $0.2 S D$ & $0.3 S D$ & $0.5 S D$ & S.E.M & MDC90 & MDC95 & $0.2 S D$ & $0.3 S D$ & $0.5 \mathrm{SD}$ \\
\hline RS-SC-10 & 3.28 & 7.66 & 9.10 & 1.70 & 2.54 & 4.24 & 3.74 & 8.72 & 10.35 & 1.81 & 2.72 & 4.53 \\
\hline RS-SC-25 & 8.01 & 18.70 & 22.21 & 3.78 & 5.67 & 9.45 & 10.35 & 24.15 & 28.68 & 4.75 & 7.12 & 11.87 \\
\hline
\end{tabular}


indicator of $0.5 \mathrm{SD}$, the mean MCIDs of RS-SC-10 and RS-SC-25 were \pm 3.95 and \pm 9.92 , respectively. Other information on distribution-based indicators is given in Table 4. As for anchor-based estimates of MCID, EORTC QLQ-C30, CD-RISC, and HADS were applied as anchors owing to their strong correlation with RS-SCs (correlation coefficient $>0.3$ ). As shown in Table 5, for patients with recovery status (improvement), linear regression estimates of the MCIDs ranged from 3.05 to 3.62 for RS-SC-10, and 8.56 to 9.31 for RS-SC-25. ROC consistently showed that $\mathrm{a}+2.0$-point change in RS-SC-10 had the best discriminant, with AUC ranging from 0.67 to 0.73 , and a +5.5 -point change in RS-SC- 25 had the best discriminant, with AUC ranging from 0.65 to 0.70 . For patients with worsening status (deterioration), linear regression estimates of the MCID ranged from -2.61 to -2.26 for RS-SC-10 and ranged from -7.05 to -6.19 for RS-SC-25. ROC consistently showed that a -1.5 -point change in RS-SC-10 had the best discriminant, with AUC ranging from 0.66 to 0.74 , and a-4.5-point change in RS-SC-25 had the best discriminant, with AUC ranging from 0.66 to 0.73 . All estimates of the MCIDs for RS-SCs are outlined in Table 6 .

\section{Discussion}

A prospective study was performed to assess the MCIDs of RS-SCs using distribution- and anchor-based methods. The findings for RS-SCs were robust as regards the choice of comparator instrument based on the large sample (623 paired RS-SCs scores) and high response rates (84.1\%). It demonstrated the convergent validity of the RS-SCs based on the significant correlations with established disease-specific scales (anchors) at baseline. In general, the correlations between RS-SC-10 and established scales were stronger than those between RS-SC-25 and other external indicators, indicating that RS-SC-10 would more likely be of interest to researchers in patients with cancer. Also, it meant that a two-factor structure was better to capture resilience characteristics than the original five-factor one, and the theoretical framework of resilience in cancer patients based on RS-SCs should be revised and redefined in future studies. In addition, we found the significant correlations between changes in RS-SCs and in anchors, providing implications for the design of resilience-related clinical intervention trials [12, 13], especially for sample size calculation; for example, a correlation coefficient of 0.31 between changes in QoLGHS and in RS-SCs was identified at T1, and as such, 132 paired measurements should be recruited to achieve $90 \%$ power and a 0.05 significance level with an anticipated dropout of $20 \%$ [37].

Although the determination of MCID remains controversial and researchers have not established consensus and methods to determine the MCID of patient-reported outcome measures, it is important in the validation of clinical instrument studies [38]. In relation to this, two

Table 5 Anchor-based MCID and ROC estimates for RS-SC at 3-month $\left(T_{1}, N=643\right)$

\begin{tabular}{|c|c|c|c|c|c|c|c|c|c|c|}
\hline Indicators & Scale & Anchors & Mean change $(95 \% \mathrm{Cl})$ & Effect Size & Cut-off & Sen & Spe & YI & AUC & $P$ \\
\hline \multirow[t]{8}{*}{ amprovement } & RS-SC-10 & QoL GHS & 3.62 (3.34 to 3.91) & 0.48 & 2.00 & 0.62 & 0.69 & 0.31 & 0.68 & $<0.001$ \\
\hline & & CD-RISC-10 & 3.29 (3.10 to 3.48$)$ & 0.41 & 2.00 & 0.71 & 0.69 & 0.40 & 0.73 & $<0.001$ \\
\hline & & HADS-A & 3.05 (2.79 to 3.20$)$ & 0.40 & 2.00 & 0.61 & 0.66 & 0.27 & 0.67 & $<0.001$ \\
\hline & & HADS-D & 3.42 (3.18 to 3.66$)$ & 0.44 & 2.00 & 0.69 & 0.64 & 0.33 & 0.69 & $<0.001$ \\
\hline & $\mathrm{RS}-\mathrm{SC}-25$ & QoL GHS & 9.31 (8.83 to 9.81) & 0.51 & 5.50 & 0.58 & 0.67 & 0.25 & 0.65 & $<0.001$ \\
\hline & & CD-RISC-10 & 8.56 (8.23 to 8.91) & 0.41 & 5.50 & 0.72 & 0.65 & 0.37 & 0.70 & $<0.001$ \\
\hline & & HADS-A & 8.78 (8.29 to 9.29) & 0.44 & 5.50 & 0.59 & 0.67 & 0.26 & 0.66 & $<0.001$ \\
\hline & & HADS-D & 8.97 (8.50 to 9.44) & 0.48 & 5.50 & 0.70 & 0.61 & 0.31 & 0.68 & $<0.001$ \\
\hline \multirow[t]{8}{*}{ beterioration } & RS-SC-10 & QoL GHS & $-2.59(-2.79$ to -2.39$)$ & -0.35 & -1.50 & 0.64 & 0.68 & 0.32 & 0.69 & $<0.001$ \\
\hline & & CD-RISC-10 & $-2.26(-2.39$ to -2.14$)$ & -0.28 & -1.50 & 0.71 & 0.71 & 0.42 & 0.74 & $<0.001$ \\
\hline & & HADS-A & $-2.61(-2.77$ to -2.43$)$ & -0.34 & -1.50 & 0.60 & 0.66 & 0.26 & 0.66 & $<0.001$ \\
\hline & & HADS-D & $-2.48(-2.65$ to -2.32$)$ & -0.32 & -1.50 & 0.74 & 0.67 & 0.41 & 0.72 & $<0.001$ \\
\hline & RS-SC-25 & QoL GHS & $-6.81(-7.18$ to -6.42$)$ & -0.37 & -4.50 & 0.61 & 0.68 & 0.29 & 0.68 & $<0.001$ \\
\hline & & CD-RISC-10 & $-6.19(-6.64$ to -5.93$)$ & -0.29 & -4.50 & 0.72 & 0.69 & 0.41 & 0.73 & $<0.001$ \\
\hline & & HADS-A & $-7.05(-7.39$ to -6.70$)$ & -0.36 & -4.50 & 0.59 & 0.68 & 0.27 & 0.66 & $<0.001$ \\
\hline & & HADS-D & $-6.79(-7.11$ to 6.47$)$ & -0.36 & -4.50 & 0.72 & 0.64 & 0.36 & 0.70 & $<0.001$ \\
\hline
\end{tabular}

Sen Sensitivity, Spe Specificity, YI Youden Index, AUC area under curve or C-statistic

a Improvement was defined for QoL GHS (raw score, increased by 1 point), CD-RISC-10 (increased by 3 points), HADS-A (decreased by 1.5 points) and HADS-D (decreased by 1.5 points)

b Deterioration was defined for QoL GHS (raw score, decreased by 1 point in global health status), CD-RISC-10 (decreased by 3 points), HADS-A (increased by 1.5 points) and HADS-D (increased by 1.5 points) 
Table 6 Summary of anchor-based and distribution-based estimates for the MCID of RS-SCs

\begin{tabular}{|c|c|c|c|c|}
\hline \multirow[t]{2}{*}{ Scale } & \multirow[t]{2}{*}{ Approach } & \multirow[t]{2}{*}{ Anchor/method } & \multicolumn{2}{|l|}{ MCID estimate } \\
\hline & & & Improvement & Deterioration \\
\hline \multirow[t]{10}{*}{ RS-SC-10 } & Distribution & S.E.M & $3.51^{\mathrm{a}}$ & $-3.51^{\mathrm{a}}$ \\
\hline & Distribution & $0.5 \mathrm{SD}$ & $4.39^{\mathrm{a}}$ & $-4.39^{\mathrm{a}}$ \\
\hline & Linear regression & QoL GHS & 3.62 & -2.59 \\
\hline & Linear regression & CD-RISC-10 & 3.29 & -2.26 \\
\hline & Linear regression & HADS-A & 3.05 & -2.61 \\
\hline & Linear regression & HADS-D & 3.42 & -2.48 \\
\hline & $\mathrm{ROC}$ & QoL GHS & 2.00 & -1.50 \\
\hline & $\mathrm{ROC}$ & CD-RISC-10 & 2.00 & -1.50 \\
\hline & $\mathrm{ROC}$ & HADS-A & 2.00 & -1.50 \\
\hline & $\mathrm{ROC}$ & HADS-D & 2.00 & -1.50 \\
\hline \multirow[t]{10}{*}{ RS-SC-25 } & Distribution & S.E.M & $9.18^{a}$ & $-9.18^{a}$ \\
\hline & Distribution & $0.5 \mathrm{SD}$ & $10.66^{a}$ & $-10.66^{a}$ \\
\hline & Linear regression & QoL GHS & 9.31 & -6.81 \\
\hline & Linear regression & CD-RISC-10 & 8.56 & -6.19 \\
\hline & Linear regression & HADS-A & 8.78 & -7.05 \\
\hline & Linear regression & HADS-D & 8.97 & -6.79 \\
\hline & $\mathrm{ROC}$ & QoL-total & 5.50 & -4.50 \\
\hline & $\mathrm{ROC}$ & CD-RISC-10 & 5.50 & -4.50 \\
\hline & $\mathrm{ROC}$ & HADS-A & 5.50 & -4.50 \\
\hline & $\mathrm{ROC}$ & HADS-D & 5.50 & -4.50 \\
\hline
\end{tabular}

a Mean

main approaches, distribution- and anchor-based methods, were both performed in this study. Distributionbased methods rely on the distribution of the cohort population and the reliability of the instrument instead of the patients' perspective. A range of different estimates of the MCID have been proposed, including SEM, 0.5 SD, and $\mathrm{MDC}_{90}$, leading to inconsistencies and significantly limited clinical interpretation [39]. As expected, the distribution-based estimates of $0.5 \mathrm{SD}$ and SEM were similar to the anchor-based estimates in the current study. However, the $\mathrm{MDC}_{90}$ and $\mathrm{MDC}_{95}$ estimates for MCID were much higher compared with other distributionor anchor-based estimates because the MDC estimates took SD, SEM, and CI into consideration [39]. In addition, we found that the distribution-based estimates of MCID were consistently greater than the anchor-based estimates, indicating a wide distribution of RS-SCs in the cohorts. Anchor-based methods, which take information on patient-reported benefit or deterioration into account, are often preferred to distribution-based methods, based on the comparison of the changes in outcomes of interest with established outcomes of change. However, no consensus has been reached as regards the threshold strength of the correlations between the outcome of interest with other anchors, and patient-reported outcomes recorded before and after interventions are subject to recall bias, which will also affect the strength of the correlations [40]. Therefore, sensitivity and specificity analyses were performed at the individual level (within subject) in our study. The MCID estimations for RS-SC-10 and RS-SC-25 yielded approximately +2.0 and +5.5 for improvement and -1.5 and -4.5 for deterioration, respectively, indicating that the RS-SCs were more responsive to the worsening status of patients with cancer. For example, a patient's RS-SC-25 decreasing by -4.5 or increasing by +5.5 would suggest that the health state of resilience has clinically changed and would be associated with other psychosocial functions (i.e. anxiety and depression). The same applies for RS-SC-10. We chose anchor-based estimates over distribution-based ones for two reasons. First, distribution-based estimates cannot directly measure MCIDs although they provide indirect and supportive information with regard to significant changes in the instrument; this view is shared by other investigators [41]. Second, the MCIDs estimated by distribution-based approaches are much greater than the mean change identified by anchor-based methods, indicating that distribution-based estimates may overestimate the true MCIDs [38, 39]. At last, RS-SCs may have potential application in resilience-based evaluation (ie, instruments and theoretical development) [40,43] and intervention for adolescents with cancer with cancer and 
their caregivers [44-47], for example, the parents of children with cancer, which is receiving enhanced interest in recent cancer research $[48,49]$. More research for this vulnerable group are urgently warranted.

\section{Limitations}

The current study has a number of limitations to be noted. First, owing to deteriorating health status or other reasons for loss to follow-up, a possible selection bias may be identified in RS-SCs, which is not obtained for ethical reasons, and may overestimate or underestimate the strength of correlations [50]. Second, there exists no golden standard for defining and measuring resilience in patients with cancer and we had to choose diseasespecific and established instruments as anchors. Thus, the findings in the current study, especially those on the differences between improvement and deterioration in RS-SCs, should be interpreted with caution and must be validated in future research. Third, the floor and ceiling effects of outcome measures should also be noted, which could indicate no room for further improvement or deterioration in health status. The current study could not address whether MCIDs for RS-SCs hold true in populations at extremes of the health spectrum (i.e. a baseline RS-SC-10 of 10 or 50 ).

\section{Conclusion}

The most reliable MCID is around 5 points for RS-SC25 and 2 points for RS-SC-10. RS-SCs are more responsive to the worsening status of resilience in patients with cancer and these estimates could be useful in future resilience-based intervention trials.

\section{Supplementary information}

Supplementary information accompanies this paper at https://doi. org/10.1186/s12955-020-01631-6.

Additional file 1: Table S1. Resilience Scale Specific to Cancer (RS-SC, English Version). S2 Table. 10-item Resilience Scale Specific to Cancer (RS-SC-10, English Version).

\section{Abbreviations}

MCID: Minimum Clinical Important Difference; RS-SC-10: 10-item Resilience Scale Specific to Cancer; RS-SC-25: 25-item Resilience Scale Specific to Cancer: EORTC QLQ-C30: European Organization for Research and Treatment of Cancer Quality of Life Questionnaire; CD-RISC: Connor-Davidson Resilience Scale; HADS: Hospital Anxiety and Depression Scale; ALI: Allostatic Load Index; SEM: Standard Error of Measurement; MDC: Minimal Detectable Change; AUC : Area Under Curve; QoL: Quality of Life; GHS: Global Health Status; PF: Physical Function; RF: Role Function; EF: Emotion Function; CF: Cognitive Function; SF: Social Function; Sen: Sensitivity; Spe: Specificity; Yl: Youden Index.

\section{Acknowledgements}

The authors acknowledge the valuable information provided by the patients who participated in the Be Resilient to Cancer program.

\section{Authors' contributions}

Zeng Jie Ye: Dr. Ye conceptualized and designed the study, carried out the initial analyses, supervised data collection, drafted the initial manuscript, and approved the final manuscript as submitted. Zhang Zhang: Dr. Zhang conceptualized and designed the study, supervised data collection, drafted the initial manuscript, and approved the final manuscript as submitted. Ying Tang: Dr. Tang supervised data collection, critically reviewed the manuscript, drafted the initial manuscript and approved the final manuscript as submitted. Jian Liang: Dr. Liang coordinated data collection, critically reviewed the manuscript, and approved the final manuscript as submitted. Xiao Ying Zhang: Dr. Zhang coordinated data collection, critically reviewed the manuscript and approved the final manuscript as submitted. Guang Yun Hu: Miss Hu coordinated data collection, critically reviewed the manuscript and approved the final manuscript as submitted. Zhe Sun: Dr. Sun coordinated data collection, critically reviewed the manuscript and approved the final manuscript as submitted. Mu Zi Liang: Dr. Liang coordinated data collection, critically reviewed the manuscript and approved the final manuscript as submitted. Yuan Liang Yu: Mr. Yu coordinated data collection, critically reviewed the manuscript and approved the final manuscript as submitted.

\section{Funding}

This research was funded by grants from National Natural Science Foundation of China (No.71904033), Humanity and Social Science Youth Foundation of Ministry of Education of China (No.19YJCZH227), Humanity and Social Science Foundation of Department of Education of Guangdong Province (No.2020WTSCX009), Humanity and Social Science Foundation of Guangzhou University of Chinese Medicine (No.2020SKXK01), Medical Scientific Research Foundation of Guangdong Province (No.A2019484), Research Fund for Talented Scholars of Guangzhou University of Chinese Medicine (No. A1-AFD018), and Innovative Project of Guangzhou University of Chinese Medicine (No.2016KYTD08).

\section{Availability of data and materials}

The datasets used and/or analyzed during the current study are available from the corresponding author on reasonable request.

\section{Ethics approval and consent to participate}

All procedures performed in studies involving human participants were in accordance with the ethical standards of the institutional and/or national research committee and with the 1964 Helsinki declaration and its later amendments or comparable ethical standards. Informed consent was obtained, and the Human Research Ethics Committee approved the present study (registration number: 2016KYTD08).

\section{Consent for publication}

All authors approved the final manuscript as submitted and agree to be accountable for all aspects of the work.

\section{Competing interests}

The authors declare that they have no conflicts of interest to this work.

\section{Author details}

${ }^{1}$ Guangzhou University of Chinese Medicine, Guangzhou 510006, Guangdong Province, China. ${ }^{2}$ Sun Yat-sen University Cancer Center, State Key Laboratory of Oncology in South China, Collaborative Innovation Center for Cancer Medicine, Guangzhou 510060, Guangdong Province, China. ${ }^{3}$ Institute of Tumor, Guangzhou University of Chinese Medicine, Guangzhou 510006, Guangdong Province, China. ${ }^{4}$ Guangdong Provincial Key Laboratory of New Drug Development and Research of Chinese Medicine, Mathematical Engineering Academy of Chinese Medicine, Guangzhou University of Chinese Medicine, Guangzhou 510006, Guangdong Province, China. ${ }^{5}$ The Seventh Affiliated Hospital, Sun Yat-sen University, Shenzhen 510275, Guangdong Province, China. ${ }^{6}$ Army Medical University, Chongqing Municipality 400038, China. ${ }^{7}$ The First Affiliated Hospital, Guangzhou University of Chinese Medicine, Guangzhou 510405, Guangdong Province, China. ${ }^{8}$ Guangdong Academy of Population Development, Guangzhou 510600, Guangdong Province, China.

${ }^{9}$ South China University of Technology, Guangzhou 510641, Guangdong Province, China. 
Received: 29 June 2020 Accepted: 23 November 2020

Published online: 09 December 2020

\section{References}

1. Chen W, Zheng R, Baade PD, et al. Cancer statistics in China, 2015. CACancer J Clin. 2016;66(2):115-32

2. McCorkle R, Ercolano E, Lazenby M, et al. Self-management: enabling and empowering patients living with cancer as a chronic illness. CA Cancer J Clin. 2011:61(1):50-62.

3. Rutter M. Resilience in the face of adversity: protective factors and resistance to psychiatric disorder. Brit J Psychiatry. 1985;147(6):598-611.

4. Ye ZJ, Qiu HZ, Li PF, et al. Predicting changes in quality of life and emotional distress in Chinese patients with lung, gastric, and colon-rectal cancer diagnoses: the role of psychological resilience. Psycho-oncology. 2017:26(6):829-35.

5. Luo D, Eicher M, White K. Individual resilience in adult cancer care: a concept analysis. Int J Nurs Stud. 2020;102:103467. Epub ahead of print. https://doi.org/10.1016/j.ijnurstu.2019.103467.

6. Ye ZJ, Liang MZ, Li PF, et al. New resilience instrument for patients with cancer. Qual Life Res. 2018;27(2):355-65.

7. Ye ZJ, Peng CH, Zhang HW, et al. A biopsychosocial model of resilience for breast Cancer: a preliminary study in mainland China. Eur J Oncol Nurs. 2018;36:95-102

8. Ye ZJ, Liang MZ, Zhang HW, et al. Psychometric properties of the Chinese version of resilience scale specific to Cancer: an item response theory analysis. Qual Life Res. 2018;27(6):1635-45.

9. Ye ZJ, Zhang Z, Tang Y, et al. Development and psychometric analysis of the 10-item resilience scale specific to cancer: a multidimensional item response theory analysis. Eur J Oncol Nurs. 2019:41:64-71.

10. Revicki D, Hays RD, et al. Recommended methods for determining responsiveness and minimally important differences for patient-reported outcomes. J Clin Epidemiol. 2008;61(2):102-9.

11. Turner D, Schünemann HJ, Griffith LE, et al. The minimal detectable change cannot reliably replace the minimal important difference. J Clin Epidemiol. 2010;63(1):28-36.

12. Ye ZJ, Liang MZ, Qiu HZ, et al. Effect of a multidiscipline mentor-based program, be resilient to breast Cancer (BRBC), on female breast cancer survivors in mainland China-a randomized, controlled, theoreticallyderived intervention trial. Breast Cancer Res Treat. 2016;158(3):509-22

13. Ye ZJ, Zhang Z, Zhang XY, et al. Effectiveness of adjuvant supportiveexpressive group therapy for breast cancer. Breast Cancer Res Tr. 2020;180(1):121-34

14. Ye ZJ, Qiu HZ, Liang MZ, et al. Effect of a mentor-based, supportiveexpressive program, be resilient to breast Cancer, on survival in metastatic breast cancer - a randomized, controlled intervention trial. $\mathrm{Br} J$ Cancer. 2017;117(10):1486-94.

15. Ye ZJ, Zhang Z, Zhang XY, et al. State or trait? Measuring resilience by generalisability theory in breast cancer. Eur J Oncol Nurs. 2020;46:101727.

16. EORTC Quality of Life Group. Quality of life research within the EORTC the EORTC QLQ-C30. Eur J Cancer. 2002;38(4):125-33.

17. Wan $\mathrm{CH}$, Chen $\mathrm{MQ}$, Zhang $\mathrm{CZ}$, et al. The introduction of the Chinese version of EORTC QLQ-C30 in patients with cancer. J Pract Oncol. 2005;4:353-5

18. Yu XN, Zhang JX. Factor analysis and psychometric evaluation of the Connor-Davidson resilience scale (CD-RISC) with Chinese people. Soc Behav Pers. 2007;35(1):19-31.

19. Ye ZJ, Qiu HZ, Li PF, et al. Validation and application of the Chinese version of the 10-item Connor-Davidson resilience scale (CD-RISC-10) among parents of children with Cancer diagnosis. Eur J Oncol Nurs. 2017:27:36-44.

20. Ni MY, Li TK, Yu NX, et al. Normative data and psychometric properties of the Connor-Davidson resilience scale (CD-RISC) and the abbreviated version (CD-RISC2) among the general population in Hong Kong. Qual Life Res. 2016;25(1):111-6.

21. Li Q, Lin Y, Hu C, et al. The Chinese version of hospital anxiety and depression scale: psychometric properties in Chinese cancer patients and their family caregivers. Eur J Oncol Nurs. 2016;25:16-23.
22. Seeman TE, McEwen BS, Rowe JW, et al. Allostatic load as a marker of cumulative biological risk: MacArthur studies of successful aging. Proc Natl Acad Sci U S A. 2001:98:4770-5.

23. Karlamangla AS, Singer BH, Seeman TE. Reduction in allostatic load in older adults is associated with lower all-cause mortality risk: MacArthur studies of successful aging. Psychosom Med. 2006;68:500-7.

24. Qiu HZ, Ye ZJ, Liang MZ, et al. Effect of an art brut therapy program, go beyond the schizophrenia (GBTS),on prison inmates with schizophrenia in mainland China-A randomized, longitudinal, and controlled trial. Clin Psychol Psychother. 2017;24(5):1069-78.

25. Ye ZJ, Cai RQ, Liu ML, et al. The development of the transitional care model in nursing in mainland China: a literature review. Int J Nurs Sci. 2016;3(1):113-30

26. DeVellis RF. Scale development : theory and applications. Thousand Oaks: SAGE; 2012.

27. Polit DF, Beck CT. Essentials of nursing research: appraising evidence for nursing practice. Philadelphia: Wolters Kluwer Health; 2018.

28. Hawkins DL, Using U. Statistics to derive the asymptotic distribution of Fisher's Z statistic. Am Stat. 1989;43(4):235-7.

29. Sokal RR, Rohlf FJ. Biometry. The principles and practice of statistics in biological research. 3rd ed. New York: WH Freeman; 1995.

30. Cohen J. Statistical power analysis for the behavioral sciences. 2nd ed. Hillsdale: Lawerence Erlbaum Associates, Inc.; 1988.

31. Norman GR, Sloan JA, Wyrwich KW. Interpretation of changes in health-related quality of life: the remarkable universality of half a standard deviation. Med Care. 2003;41:582-92.

32. Wyrwich KW, Tierney WM, Wolinsky FD. Further evidence supporting an SEM-based criterion for identifying meaningful intraindividual changes in health-related quality of life. J Clin Epidemiol. 1999;52:861-73.

33. Raman S, Ding K, Chow E, et al. Minimal clinically important differences in the EORTC QLQ-C30 and brief pain inventory in patients undergoing reirradiation for painful bone metastases. Qual Life Res. 2018;27(4):1089-98.

34. Ye ZJ, Wang ZY, Yu YL, Liang MZ, et al. Reliability and validity of the Chinese version of the 10-item Connor-Davidson resilience scale in Chinese Cancer patients. Chin Gen Pract. 2018;21(15):1839-44.

35. Smid DE, Franssen FM, Houben-Wilke $S$, et al. Responsiveness and MCID estimates for CAT, CCQ, and HADS in patients with COPD undergoing pulmonary rehabilitation: a prospective analysis. J Am Med Dir Assoc. 2017;18(1):53-8.

36. Youden WJ. Index for rating diagnostic tests. Cancer. 1950;3(1):32-5

37. Zar JH. Biostatistical analysis. 5th ed. Englewood Cliffs: Prentice-Hall; 2009.

38. Kon SS, Canavan JL, Jones SE, et al. Minimum clinically important difference for the COPD assessment test: a prospective analysis. Lancet Respir Med. 2014;2:195-203.

39. Copay AG, Subach BR, Glassman SD, et al. Understanding the minimum clinically important difference: a review of concepts and methods. Spine J. 2007;7:541-6

40. Schunemann HJ, Griffith $L$, Jaeschke R, et al. Evaluation of the minimal important difference for the feeling thermometer and the St. George's respiratory questionnaire in patients with chronic air flow obstruction. J Clin Epidemiol. 2003;56:1170-6.

41. Puhan MA, Chandra D, Mosenifar Z et al. The minimal important difference of exercise tests in severe COPD. Eur Respir J. 2011;37:784-90.

42. Ye ZJ, Qiu HZ, Li PF, et al. Resilience model for parents of children with cancer in mainland China-an exploratory study. Eur J Oncol Nurs. 2017;27:9-16

43. Ye ZJ, Liu ML, Zhang Z, et al. Psychometric properties of the Chinese version of the parent perception of uncertainty scale (PPUS) among parents of children with cancer diagnosis. Intl J Nurs Sci. 2017:4(3):278-84.

44. Rosenberg AR, Bradford MC, Junkins CC, et al. Effect of the promoting resilience in stress management intervention for parents of children with Cancer (PRISM-P): a randomized clinical trial. JAMA Netw Open. 2019:2(9):e1911578.

45. Yi-Frazier JP, Fladeboe K, Klein V, et al. Promoting resilience in stress Management for Parents (PRISM-P): an intervention for caregivers of youth with serious illness. Fam Syst Health. 2017;35(3):341-51.

46. Ye ZJ, Zhang Z, Liang MZ, et al. Symptoms and management of children with incurable cancer in mainland China. Eur J Oncol Nurs. 2019;38:42-9.

47. Rosenberg AR, Bradford MC, McCauley E, Curtis JR, Wolfe J, Baker KS, Yi-Frazier JP. Promoting resilience in adolescents and young adults with 
cancer: results from the PRISM randomized controlled trial. Cancer. 2018;124(19):3909-17.

48. Ye ZJ, Cheng MH, Zhang XY, et al. Treatment Decision-Making and Regret in Parents of Children with Incurable Cancer. Cancer Nurs, 2019, online, doi:https://doi.org/10.1097/NCC.00000000000000783.

49. Ye ZJ, Guan HJ, Wu LH, et al. The resilience and psychosocial function among mainland Chinese parents of children with cancer: a cross-sectional survey. Cancer Nurs. 2015;38(6):466-74.
50. Ye ZJ, Zhang XY, Liang J, et al. The challenges of medical ethics in China: are gene-edited babies enough? Sci Eng Ethics. 2020;26(1):123-5

\section{Publisher's Note}

Springer Nature remains neutral with regard to jurisdictional claims in published maps and institutional affiliations.
Ready to submit your research? Choose BMC and benefit from:

- fast, convenient online submission

- thorough peer review by experienced researchers in your field

- rapid publication on acceptance

- support for research data, including large and complex data types

- gold Open Access which fosters wider collaboration and increased citations

- maximum visibility for your research: over $100 \mathrm{M}$ website views per year

At BMC, research is always in progress.

Learn more biomedcentral.com/submissions 